\title{
Hypoxic Oxygen Fluctuations Produce Less Severe Retinopathy than Hyperoxic Fluctuations in a Rat Model of Retinopathy of Prematurity
}

\author{
JANET R. MCCOLM, STEVE CUNNINGHAM, JEAN WADE, KOFI SEDOWOFIA, \\ BALAZS GELLEN, TARUN SHARMA, NEIL MCINTOSH, AND BRIAN W. FLECK \\ Child Life and Health, Reproductive and Developmental Sciences, University of Edinburgh, Edinburgh, \\ EH9 1UW, United Kingdom [J.R.M., J.W., K.S., B.G., N.M.]; Royal Hospital for Sick Children, \\ Edinburgh, EH9 ILF, United Kingdom [S.C.]; Princess Alexandra Eye Pavilion, Edinburgh, EH3 9HA, \\ United Kingdom [T.S., B.W.F.]
}

\begin{abstract}
The aim of this study was to investigate whether the mean around which arterial oxygen fluctuations take place was important in a unique animal model of oxygen-induced retinopathy. Retinopathy of prematurity (ROP) is associated with fluctuating arterial oxygen. A recent retrospective study suggested that management of high-risk preterm infants at lower oxygen saturations was associated with less severe ROP. Rat pups were raised in a variable oxygen environment around a high (24\%), normal $(21 \%)$ or low $(17 \%)$ mean inspired oxygen for $14 \mathrm{~d}$. Rat pups raised in the high (24\%) mean variable oxygen environment had more retarded retinal vascular development than did rats raised in an environment that fluctuated around $21 \%$ mean oxygen. In contrast, rats raised in a lower mean (17\%) but still variable oxygen environment had no discernible retinal differences from controls raised in constant room air. Rats raised in a relatively hypoxic but variable oxygen environment develop less
\end{abstract}

\section{ABSTRACT}

severe retinal vascular abnormalities than those raised in variable oxygen around higher oxygen means. (Pediatr Res 55: 107-113, 2004)

$\quad$ Abbreviations
C, group raised in room air
DAB, diaminobenzidine
Hi, variable oxygen profile around $24 \%$ oxygen mean
IQR, interquartile range
Lo, variable oxygen profile around $17 \%$ oxygen mean
N, variable oxygen profile around $21 \%$ oxygen mean
PFA, paraformaldehyde
ROP, retinopathy of prematurity
TBS, Tris-buffered saline
VEGF, vascular endothelial growth factor

ROP is a leading cause of blindness among infants in developed (1) and middle-income countries (2). The role of high concentrations of supplemental oxygen was first recognized in the 1950s (3), and subsequent animal studies (4-6) delineated the pathophysiology. Restrictions on oxygen use led to a reduction in the disease, but the technological advances in the 1970 s and 1980 s led to infants up to $16 \mathrm{wk}$ preterm surviving. Even with strict oxygen control to prevent hyperoxia the disease incidence began to increase again. The cause of the current disease is generally considered multifactorial (7), how-

Received September 19, 2002; accepted June 11, 2003.

Correspondence: Janet McColm, Ph.D., Department of Ophthalmology, University of North Carolina, 6135 Neuroscience Research Building, 103 Mason Farm Road CB\#7041, Chapel Hill, NC 27599-7041, U.S.A.; e-mail: jmccolm@email.unc.edu

Supported by grants from the Royal Blind School Edinburgh, United Kingdom; Mason Medical Foundation Brighton, United Kingdom; Ross Foundation for the Prevention of Blindness Edinburgh, United Kingdom; Research into Eye Disease Trust, London, United Kingdom.

J.R.M. and S.C. contributed equally to this article.

DOI: 10.1203/01.PDR.0000099772.66376.02 ever variations in oxygen have been shown important to the development of disease in humans $(8,9)$ and in animal models $(10-12)$.

Current oxygen therapy for preterm neonates in our neonatal unit (Edinburgh, UK) provides a "safe" transcutaneous range of $6-10 \mathrm{kPa}(45-75 \mathrm{~mm} \mathrm{Hg})$. This is considered to minimize hyperoxia-associated ROP and to limit neurologic and pulmonary morbidity from associated hypoxia. However, there are few data to indicate whether these limits are appropriate. In "healthy" preterm infants (without lung disease) of 29-34 wk gestation, Mok et al. (13) demonstrated a mean (range) of 9 $\mathrm{kPa}(4.7-10.9 \mathrm{kPa})$ [67.5 (35-82) $\mathrm{mm} \mathrm{Hg}$ ] over the first few months of life. However, no data are available for the gestations at risk for ROP currently (infants $<29$ wk gestation).

A recent retrospective case review suggested that babies $<28$ wk gestational age nursed in an oxygen saturation range of $70-90 \%$ had a lower incidence of ROP (14) when compared with infants nursed in a higher range 88-98\%. Infants nursed in the lower oxygen saturation range had no increased inci- 
dence of cerebral palsy or adverse pulmonary outcomes at routine follow-up, however, detailed long-term follow-up was not performed. A review of practice in the United States (15) also showed that the incidence of ROP in neonatal intensive care units where oxygen saturations were maintained at a maximum of $92 \%$ was less than half that in units where the oxygen saturation was greater than $92 \%$.

The study of ROP pathophysiology in humans is ethically and technically difficult and much of our recent understanding is derived from animals (16). Animal models have, until recently, poorly represented the oxygen environment experienced by an extremely preterm infant $(11,17,18)$ partly due to technical difficulties in recreating the fluctuations that are seen in the preterm infant. We have developed a system, in conjunction with BioSpherix (Redfield, NY, U.S.A.) which can make accurate changes of up to $50 \%$ oxygen within $1 \mathrm{~min}$ in a rat cage (19). This equipment has enabled us to demonstrate that the oxygen fluctuations experienced by a preterm infant who developed severe ROP could also produce oxygeninduced retinopathy in rats exposed to similar clinically relevant oxygen fluctuations around a normoxic [fraction of inspired oxygen $\left(\mathrm{FiO}_{2}\right) 21 \%$ ] mean (12). The aim of the current study was to assess whether oxygen-induced retinopathy could be influenced by small changes in the mean oxygen concentration to either a relatively hyperoxic mean or relatively hypoxic mean, while maintaining the same oxygen variability profile.

\section{METHODS}

\section{Animal Model}

This was a group-controlled study where at least three litters of 12 pups each were assigned per experimental or control group. Samples were masked before assessment. The UK Home Office gave approval for this study, and all animals were cared for in accordance with UK Home Office legislation and the Association for Research in Vision and Ophthalmology Statement for the Use of Animals in Vision and Ophthalmic Research.

Derivation of the oxygen profiles. The Neonatal Intensive Care Unit in Edinburgh has a computerized physiologic monitoring system that has gathered data from admitted infants since 1990 (20). Arterial oxygen is continuously monitored transcutaneously and a mean of 601 -s data points are stored each minute. Our previous study had shown that infants with greater variability in their arterial oxygen had more severe disease (8). Therefore, the computerized transcutaneous oxygen profile representing partial pressure of arterial oxygen of one infant who developed severe (threshold) retinopathy of prematurity was selected, and the first $14 \mathrm{~d}$ used as the experimental profile (21,327 data points). The infant was not a random selection, rather it was the infant with the most extreme oxygen variability from the group of 31 so as to have maximum chance of producing retinopathy should oxygen variability have been associated. We did not use pooled data from more than one infant as this would have blunted the frequent small fluctuations of transcutaneous oxygen values seen in individual infants.
Translation of infant values to equivalent rat values. Optimal arterial oxygen levels for a preterm infant are open to significant debate. For many years accepted practice in UK neonatal units has been to maintain transcutaneous oxygen values between 6 and $10 \mathrm{kPa}$ ( 45 and $75 \mathrm{~mm} \mathrm{Hg}$ ), resulting in a mean value of $8 \mathrm{kPa}(60 \mathrm{~mm} \mathrm{Hg})$, as in our index preterm infant. In the United States, similar values are also recommended (21). Neonatologists appear to assume that an average arterial oxygen of $8 \mathrm{kPa}$ is sufficient to enable satisfactory metabolism and brain development, with minimal risk of retinopathy or other oxygen injury. We adopted this assumption when deciding what level of oxygen to base as the midpoint for fluctuation in our animal model.

Rat pups have an arterial oxygen of $12.9 \mathrm{kPa}(97 \mathrm{~mm} \mathrm{Hg})$ in $21 \%$ oxygen, and so, if we had directly applied the recorded fluctuations to achieve a mean of $8 \mathrm{kPa}$, the rat pups would have been almost constantly hypoxic. As we wished to assess whether fluctuation around normoxia was important, we therefore adjusted the recorded values from the infant (with an assumption of $8 \mathrm{kPa}$ as normoxia) to be equivalent to fluctuations around the known normoxia in rats $(12.9 \mathrm{kPa})$. To every value in the infant's recorded profile we had to add $4.9 \mathrm{kPa}$ to account for the difference in the rat and infant normoxia. This gave us our "normoxic" profile $(\mathrm{N})$, which simulated the arterial fluctuations the infant experienced in the rat.

The arterial oxygen concentrations of the rat can be made to fluctuate by varying the inspired oxygen, as the relationship of inspired to arterial oxygen concentration in the rat is linear (11). Thus, by altering the inspired oxygen profile one can theoretically achieve the same blood oxygen profile in rat pups that produced severe retinopathy in the index preterm infant. We have previously shown that this is possible and have recreated changes indicative of retinopathy in rats using this profile (12).

To ensure changes in inspired oxygen were translated to the blood within $1 \mathrm{~min}$, we performed experiments in three sedated adult rats where the femoral artery was catheterized and rapidly changed the inspired oxygen that the adult rat was breathing. With blood gas analysis we found that changes of up to $5 \%$ in inspired oxygen were translated to the blood within $1 \mathrm{~min}$ (unpublished results). It is also our clinical neonatal experience that altering the inspired oxygen would result in an alteration in the arterial oxygen within $1 \mathrm{~min}$.

Altering mean arterial oxygen values while maintaining the same fluctuations. To determine the importance of the mean arterial oxygen level in contrast to oxygen fluctuations, we used the same varying oxygen profile of 21,327 1-min data points from the index preterm infant but adjusted the inspired oxygen concentrations so that the mean levels varied. The lower limit of the clinically "safe" range is $6 \mathrm{kPa}, 2 \mathrm{kPa}$ below the infants assumed normoxia of $8 \mathrm{kPa}$. To simulate a mean of $6 \mathrm{kPa}$ in the rat we therefore reduced the values from our normoxic rat profile by $2 \mathrm{kPa}$ each, thereby fluctuating the rat arterial oxygen around a mean of $10.9 \mathrm{kPa}\left(17.9 \% \mathrm{Fio}_{2}\right)$. The rat pups raised in this profile (Lo) were mildly hypoxic $(<21 \%$ $\mathrm{O}_{2}$ ) for $88.7 \%$ of the time, however, the relative fluctuations were identical to the $\mathrm{N}$ profile. With similar reasoning we used the upper limit of the clinically safe human range, $10 \mathrm{kPa}$, to 
generate a mildly hyperoxic profile from the index preterm infant. To each of the values in the $\mathrm{N}$ profile we added $2 \mathrm{kPa}$ and then generated the inspired oxygen values the rat would need to breathe to achieve these values. This resulted in a profile $(\mathrm{Hi})$, which fluctuated oxygen around $14.9 \mathrm{kPa}(24.7 \%$ $\mathrm{FiO}_{2}$ ) in the rat and in which the rat spent $91.8 \%$ of the time hyperoxic $\left(>21 \% \mathrm{O}_{2}\right)$.

The details of these profiles are given in Table 1 and a representative hour of each profile is shown in Figure 1.

Oxygen delivery system. A computer-controlled delivery system was devised and built in conjunction with BioSpherix. This system is described fully elsewhere (19), however, in brief, the system delivers either oxygen or nitrogen to cause the required atmospheric change in oxygen. It is capable of accurately producing up to a $\pm 50 \%$ change in atmospheric oxygen within $1 \mathrm{~min}$. During testing, the median difference between required and monitored value of oxygen was $0.3 \%$, IQR 0.2 $0.7 \%(n=17,465)$. Of all monitored readings, $85 \%$ were within $1 \%$ oxygen either side of the set-point; $95 \%$ were within $\pm 2 \%$ oxygen (19).

Animal groups. A control group (C) consisted of animals raised in room air. They were raised in the same room as the experimental groups but were separate litters to separate dams. Their cages were similar to experimental groups in terms of shape, size, and color, with opaque sides and bottom and a wire grid for the roof.

The three variable oxygen groups were the fluctuations around the hypoxic mean (Lo); normoxic mean $(\mathrm{N})$, and hyperoxic mean $(\mathrm{Hi})$. The normoxic group $(\mathrm{N})$ differ from the controls (C) in that $\mathrm{N}$ had fluctuations of oxygen around a mean of $21 \%$ oxygen, whereas $\mathrm{C}$ were raised in room air at a fixed $21 \%$. Pregnant animals in all groups were acclimatized to their cages at least $24 \mathrm{~h}$ before delivery. A minimum of 12 pups was required per control or experimental litter and pups from litters born on the same day were used to supplement any litters smaller than 12. Unlike other models (22), litter sizes were not artificially enlarged to 25 pups as we wished to maintain uniform physiologic conditions for litters, other than manipulating atmospheric oxygen. Litter sizes of 25 pups have a high mortality rate around $60 \%$, which makes data analysis difficult, whereas survival rates of animals in our experiments were $100 \%$. Experiments were begun $<4 \mathrm{~h}$ after the delivery of the final pup in each litter. Bedding was changed every $7 \mathrm{~d}$, at which point the profile was paused and restarted a few minutes later. No other interruption to the profile was required. In the

Table 1. Details of inspired oxygen (\%) in the three experimental oxygen profiles

\begin{tabular}{lccc}
\hline & $\begin{array}{c}\text { Hypoxic } \\
(\text { Lo })\end{array}$ & $\begin{array}{c}\text { Normoxic } \\
(\mathrm{N})\end{array}$ & $\begin{array}{c}\text { Hyperoxic } \\
(\mathrm{Hi})\end{array}$ \\
\hline$n^{*}$ & 21,327 & 21,327 & 21,327 \\
Mean $(95 \% \mathrm{CI})$ & 17.9 & 21.3 & 24.7 \\
& $(17.9-18.0)$ & $(21.2-21.3)$ & $(24.6-24.7)$ \\
Range & $5.8-38.1$ & $9.2-41.5$ & $12.6-44.9$ \\
Time $<21 \% \mathrm{O}_{2}(\%)$ & 88.7 & 52 & 8.9 \\
Time $>21 \% \mathrm{O}_{2}(\%)$ & 11.3 & 48 & 91.8 \\
\hline
\end{tabular}

*Number of inspired oxygen data points taken from the first $14 \mathrm{~d}$ of the index infant's profile.

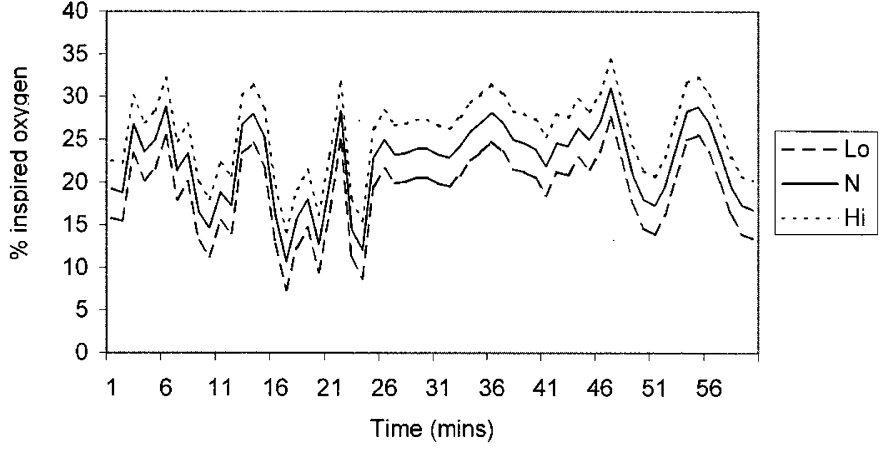

Figure 1. One hour from $\mathrm{d} 7$ of each profile. The relative fluctuation remains the same around a different mean level of oxygen for each profile.

experimental groups, carbon dioxide was maintained at normal atmospheric levels by using a relatively fast environmental turnover and incorporating soda lime within the cages.

End of the experiments. At $14 \mathrm{~d}$, experiments were stopped and the rat pups weighed. All pups were then anesthetized by intraperitoneal injection of ketamine $(2.5 \mathrm{mg} / \mathrm{kg})$ and xylazine $(1 \mathrm{mg} / \mathrm{kg})$. PFA was then directly perfused $(0.4 \mathrm{~mL} 0.5 \%)$ into the left ventricle, before euthanasia by intracardiac injection of pentobarbitone $(80 \mathrm{mg} / \mathrm{kg})$. Both eyes were enucleated; the left was prepared for cryosectioning and the right for whole mounts. The left eyes were not analyzed for this study, therefore all analyses presented here were performed on the right eyes.

\section{Histologic and Molecular Biologic Endpoints}

Retinal whole mounts. The retinas were dissected using a modification of the method of Chan-Ling (23). Enucleated eyeballs were fixed whole in 2\% PFA for $2 \mathrm{~h}$ before being washed in $1 \mathrm{M}$ PBS $\mathrm{pH}$ 7.4. Under a dissecting microscope, an incision was made at the limbus between the cornea and sclera. Scissors were then used to cut around the junction between the cornea and sclera until the cornea could be removed. The lens was gently removed, taking care not to remove the retina. The eyecup was transferred to $1 \mathrm{M}$ PBS for further dissection. The retina was gently eased from the sclera using fine forceps, taking care to leave the ora serrata intact as it defines the edge of the retina. The retina was then placed onto a TESPA(3'aminopropyltriethoxysilane) coated slide and flattened by making four incisions perpendicular to its outer edge. At this stage, as much vitreous as possible was removed using cellulose sponges and scissors.

Technically, retinal dissection is demanding and not all eyes yielded a retina that could be analyzed. We also took some eyes within each group for other staining procedures not detailed here. The final number in each group of Table 2 is the number of right eyes that we analyzed for each experimental procedure. However, we controlled for between-litter variation with our statistical analysis

Lectin stain. Endothelial cells were visualized by incubation with Griffonia simplicifolia (Bandeiraea) isolectin B4 as follows. The flattened whole-mounted retinas were made permeable in $70 \% \mathrm{vol} / \mathrm{vol}$ ethanol (kept at $-20^{\circ} \mathrm{C}$ ) for $20 \mathrm{~min}$, and then in $1 \mathrm{M} \mathrm{PBS} / 1 \%$ Triton $\mathrm{x}-100$ for $30 \mathrm{~min}$. The retinas were 
Table 2. Results of analysis from retinal wholemounts from all experimental groups

\begin{tabular}{|c|c|c|c|c|c|}
\hline & Control & $\begin{array}{l}\text { Hypoxic } \\
\text { (Lo) }\end{array}$ & $\begin{array}{l}\text { Normoxic } \\
(\mathrm{N})\end{array}$ & $\begin{array}{l}\text { Hyperoxic } \\
\text { (Hi) }\end{array}$ & $\begin{array}{l}\text { Overall Test } \\
(v s \text { control })\end{array}$ \\
\hline$n$ & 30 & 35 & 30 & 42 & \\
\hline Branches $/ \mathrm{mm}^{2}$ & $\begin{array}{c}277 \\
(253-311)\end{array}$ & $\begin{array}{c}266 \\
(226-293)\end{array}$ & $\begin{array}{l}261 \\
(215-290)\end{array}$ & $\begin{array}{l}296 \\
(267-320)\end{array}$ & $p=0.08$ \\
\hline $\begin{array}{l}\text { Peripheral avascularity } \\
\text { (\% total retina area) }\end{array}$ & $0 \%$ & $0 \%$ & $\begin{array}{r}1.7 \% \\
(0-7.9)\end{array}$ & $\begin{array}{r}2.7 \% * \\
(0.7-3.6)\end{array}$ & $\begin{aligned} p & <0.001 \\
(* p & <0.001)\end{aligned}$ \\
\hline $\begin{array}{l}\text { Weights }(\mathrm{g}) \\
\text { (mean, } 95 \% \mathrm{CI})\end{array}$ & $\begin{array}{c}29.2 \\
(28.9-29.5)\end{array}$ & $\begin{array}{c}23 * \\
(22.7-23.3)\end{array}$ & $\begin{array}{c}23.7^{*} \\
(23.5-23.9)\end{array}$ & $\begin{array}{c}25.2 \# \\
(24.3-26.1)\end{array}$ & $\begin{aligned} p & <0.05 \\
(* p & <0.001)\end{aligned}$ \\
\hline
\end{tabular}

Values are median (interquartile range) unless otherwise stated. Overall test results were from multilevel analysis and incorporated a random effect for litter as well as fixed effects for group comparisons. Likelihood ratio tests were carried out using a chi-squared approximation. All four groups were compared initially, and if this test was significant, follow-up pairwise group comparisons were made using a multiple testing correction based on the studentized range principle ( $p$ values are given in brackets where $* p<0.001$ and $\# p<0.05$ ).

then incubated with biotinylated G. simplicifolia (Bandeiraea) isolectin B4 (ICN Pharmaceuticals, Basingstoke, Hampshire, UK) at $5 \mu \mathrm{g} / \mathrm{mL}$ in $1 \mathrm{M}$ PBS overnight at $4^{\circ} \mathrm{C}$. They were rinsed in $1 \mathrm{M} \mathrm{PBS} / 1 \%$ Triton $\mathrm{x}-100$ for $10 \mathrm{~min}$, then in $1 \mathrm{M}$ PBS for 10 min twice. Streptavidin-conjugated FITC (Sigma Chemical) at $25 \mu \mathrm{g} / \mathrm{mL}$ in $1 \mathrm{M}$ PBS was added for $4 \mathrm{~h}$ at room temperature, and the slides were rinsed twice in $1 \mathrm{M}$ PBS for 10 min each. The retinas were mounted in PBS:glycerol 2:1 and the coverslip sealed with nail varnish. They were viewed using an argon/krypton laser confocal microscope (Leica, Wetzlar, Germany), which allowed low- and high-powered images to be taken and digitally stored for later analysis.

\section{Analysis of Whole Mounts}

Capillary density. Capillary bed sample areas were chosen in the central retina, with no major vessels present in the fields analyzed. As each retina has four quadrants, to allow flattening onto the slide, one area from the center of each quadrant was imaged, making four areas in total. Images were taken at $\times 100$ magnification and stored for later analysis. All stored files were assigned a random number to mask the observers and counts of the number of branches per square millimeter were made.

Avascular areas. Digitized images of the total retinal area and peripheral avascular area were measured using Scion Image Software (Scion Corporation, Frederick, MD, U.S.A.) and the avascular area was expressed as a percentage of the total retinal area.

Immunohistochemistry. Cryosections were allowed to come to room temperature and incubated for $1 \mathrm{~h}$ using biotinylated G. simplicifolia (Bandeiraea) isolectin B4 (ICN) at $12.5 \mu \mathrm{g} / \mathrm{mL}$ in TBS. After successive washes in TBS, the slides were then incubated with peroxidase-labeled streptavidin (DAKO, Bucks, UK) at $8.75 \mu \mathrm{g} / \mathrm{mL}$ for $1 \mathrm{~h}$. After washing, DAB (Sigma Chemical) was applied for $5 \mathrm{~min}$, the slides were rinsed in tap water and counterstained in hematoxylin, then mounted in Depex (VWR International Ltd., Poole, U.K.) and viewed under a light microscope. Slides were analyzed for preretinal vessels that grow out from the surface of the retina, superficial to the inner limiting membrane.

\section{Statistics}

Summary statistics are presented as mean (SD) or median (IQR). To take account of variation between litters in re- sponses, the treatments groups were compared by multilevel models using MLWin (Institute of Education, University of London, London, UK) software and incorporating a random effect for litter as well as fixed effects for group comparisons. Likelihood ratio tests were carried out using a $\chi^{2}$ approximation. All four groups were compared initially, and if this test was significant, follow-up pair-wise group comparisons were made using a multiple testing correction based on the Studentized range principle.

\section{RESULTS}

\section{Retinal Whole Mounts}

Results for all groups are given in Table 2.

Peripheral avascularity. Both the control and Lo groups were fully vascularized, whereas the $\mathrm{N}$ and $\mathrm{Hi}$ groups had a peripheral avascular area. The overall test gave a significance of $p<0.001$ and pair-wise comparisons gave a significant result for Hi versus control, whereas all other groups were not significant. Figure 2 shows a representative control $(A)$ and $\mathrm{Hi}$ $(B)$ image taken at the periphery of the endothelial cell-stained flat mount.

Capillary density. The overall four-group comparison was not significant $(p=0.08)$ so pair-wise tests were not done. However, there is a strong suggestion that the $\mathrm{N}$ and Hi groups differ, as an uncorrected pair-wise test gives $p<0.01$. Figure 2 shows a sample image of the capillary density in the control $(C)$, Hi $(D)$, and $\mathrm{N}(E)$ groups. The control group $(C)$ has a typical mature appearance, with remodeled capillary beds and avascular areas around the larger vessels (marked with a white asterisk). The Hi group $(D)$ has features of a less mature vasculature, with increased numbers of smaller vessels, and large vessels without surrounding avascular areas.

Abnormal vessels. Observers noted no extraretinal neovascularization on the flat mounts. As these are often difficult to distinguish in these preparations, serial sections were made and stained with lectin for endothelial cells. No nuclei were seen above the inner-limiting membrane of the retina in these preparations. However, two unbiased observers noted abnormal terminal dilatations present at the vascular/avascular interface of $21 \%$ of the flat-mounted retinas from the $\mathrm{N}$ group and $43 \%$ of the Hi group. These vessels were not seen in any of the retinas from the control or the Lo group. Figure $2 F$ gives 

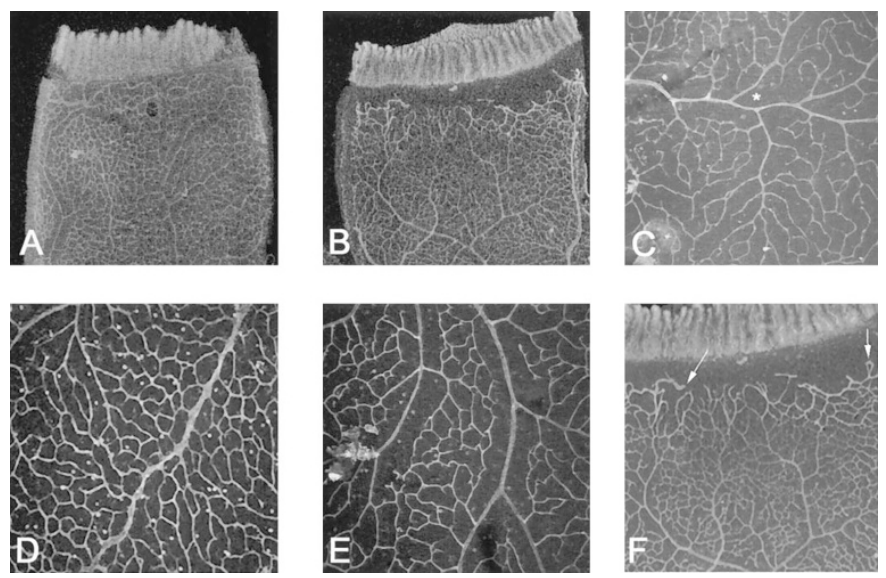

Figure 2. Endothelial cell specific lectin $[G$. simplicifolia (Bandeiraea) isolectin B4] stained retinal flat mounts $(A-F)$. Peripheral retina from room air-raised control $(A$, magnification $\times 5)$ and from experimental group $\mathrm{Hi}$ (fluctuating oxygen around a hyperoxic mean) $(B$, magnification $\times 5)$. $C, D$, and $E$ (all magnification $\times 10$ ) are all typical images from the central retinal area. Room air-raised controls $(C)$ show a typical mature capillary network that has avascular areas around the larger arterial vessels (marked with asterisk). This is in contrast to retinas from the Hi group $(D)$, which have many more capillaries than controls, which remain in a homogenous network around both arteries and veins. The group raised in oxygen fluctuating around a normoxic mean $\mathrm{N}(E)$, have slightly less central capillaries than controls, but the vasculature appears remodeled. Terminal dilations were seen in experimental groups $\mathrm{N}$ and $\mathrm{Hi}$ and a typical example of these vessels is marked with white arrows in $F$ (magnification $\times 10$ ), a higher magnification of panel $B$.

a typical example of one of these terminal dilations from the $\mathrm{Hi}$ group (marked with white arrows).

Postexperimental weights. Body weights at $14 \mathrm{~d}$ [mean ( $95 \%$ confidence intervals)] were $29.2 \mathrm{~g}(28.3,30.2 \mathrm{~g})$ in the controls, $23.7 \mathrm{~g}(23.0,24.4 \mathrm{~g})$ in the $\mathrm{N}$ group, $23 \mathrm{~g}(22.7$, $23.3 \mathrm{~g})$ in the Lo group, and $25.2 \mathrm{~g}(24.3,26.1 \mathrm{~g})$ in the $\mathrm{Hi}$ group. All three fluctuating oxygen groups had a significantly lower postexperimental body weight compared with the control group $(p<0.05)$.

\section{DISCUSSION}

Our model of retinopathy has previously demonstrated that small, frequent fluctuations in oxygen, mimicking those experienced by a human preterm, can induce features of ROP in rats (12). We now demonstrate that small shifts in mean inspired oxygen are able to influence these changes in the retinal vasculature.

Rat pups raised in a fluctuating oxygen environment in which they spent $90 \%$ of their time above $21 \%$ inspired oxygen, but in a mean oxygen concentration of only $24.7 \%$, had the most severe differences from controls. Their peripheral retina was avascular, suggesting that the retinal vascular growth had been retarded. As size of avascular area is correlated with disease severity, this avascular zone is likely to be significant $(24,25)$. The central capillary density in this group was higher than controls and showed smaller capillary-free zones around the larger vessels. The vasculature was homogenous, indicative of an immature, unremodeled vasculature (26-28), and again suggests at least a delay in the normal retinal vascular process. Finally, although no preretinal vascu- larization was seen in these pups, there were many abnormal, dilated, terminal buds at the vascular/avascular interface that were not seen in controls at any stage of vascular development. We postulate these may correlate clinically with the surface retinal new vessels seen at the ROP disease ridge shortly before extraretinal new vessels (stage 3 ROP) develop, and we are currently working to characterize these abnormal vessels.

The pups that were raised in a fluctuating oxygen environment, where $50 \%$ of the time was spent above and below $21 \%$ (mean 21.3\%) inspired oxygen, had many of the changes associated with the previously described hyperoxic variable rats, but the differences were less severe. Although there was a peripheral avascular area, it was smaller in size and there were fewer abnormal terminal vessels present. There were no differences in the central capillary density, suggesting the vasculature had been remodeled in this group and was more mature. This was confirmed by visual assessment of the flat-mounted retinas.

In contrast, the pups raised in the variable oxygen around a mildly hypoxic mean, where $90 \%$ of their time was spent below $21 \%$ oxygen (mean oxygen concentration $17.9 \%$ ), were no different from the room air-raised controls. The retinas were fully vascularized, with a vasculature that had been remodeled and appeared mature, and had no abnormal terminal vessels.

The differences in body weight between controls and experimental groups have been shown in other investigators' models of oxygen-induced retinopathy $(11,29)$. In all our groups there was no difference between litter sizes nor in the time the pups spent with their dams. We do not rule out the possibility that mothers raised in the variable oxygen environments were affected and may have passed this on to their pups indirectly, however, we did not directly measure this. We have begun to collect other tissues from the pups and their dams for analysis in an attempt to answer this issue.

These results confirm previous animal studies that hyperoxia (5) and oxygen fluctuations $(10,11)$ are important in determining disease severity. However, our model of subtle changes in inspired oxygen and clinically relevant profiles contrasts with previous animal models of ROP, which, although they may characterize severe neovascularization, do not reflect the present-day clinical management of oxygen therapy. Therefore, whether the oxygen injuries induced in previous animal models of ROP are representative of human disease, especially in terms of ROP in the 21st century, is debatable.

Our results show that fluctuations around a mildly hyperoxic mean can result in a delay of vascularization and an immature retinal vasculature. The anterior retina then remains avascular at a time when metabolic demand is increasing and local tissue ischemia may then trigger pathologic angiogenic processes.

The role of supplemental oxygen in the development and progression of ROP is still of interest. The STOP-ROP trial (30) was a recent randomized controlled trial assessing the ability of two different oxygen saturation target ranges to prevent progression of prethreshold ROP. The aim was to prevent hypoxically driven neovascularization by providing sufficient oxygen to the poorly vascularized prethreshold retina. This trial was based on a hypothesis taken from work in newborn kittens $(31,32)$ that were placed in $70-80 \%$ oxygen 
for the first few days of life and then returned to room air. Hyperoxic vasoconstriction and obliteration of the blood vessels on the retina resulted in relative retinal hypoxia, which it was hypothesized promoted hypoxically induced growth factors and subsequent vasoproliferation. If, however, the kittens were removed from hyperoxia to $28 \%$ supplemental oxygen then they had less retinal abnormalities than kittens removed from hyperoxia to room air. Overall, the STOP-ROP study failed to demonstrate any reduction in the rate of progression of moderate to severe ROP, for which there are several possible reasons. Oxygen saturation is limited in its ability to predict partial pressure of arterial oxygen, particularly in the extreme high and low values. Therefore subtle, frequent fluctuation of oxygen within the saturation limits may cause ongoing vascular instability. Preterm infants with chronic lung disease of prematurity are not stable even during the stage of neovascularization, hence it is possible that supplemental inspired oxygen may only serve to fluctuate oxygen around a higher mean. As we have shown here, the retinal vasculature is sensitive to such oxygen fluctuations. The HOPE-ROP study (33) followed infants with prethreshold ROP that did not fit the criteria for entry to the STOP-ROP trial (i.e. they had prethreshold ROP but did not require supplemental oxygen therapy). Although these infants were less likely to progress to threshold ROP than STOP-ROP infants, it is hard to determine whether their significantly greater gestational age at birth was the most significant factor. We believe from our clinical work and this current study that the "scene has been set" in the first weeks after birth, and that this is not addressed by either the STOPROP or HOPE-ROP studies.

Our studies would suggest that early manipulation may be able to improve retinal outcome, and evidence to support this is also beginning to emerge from clinical studies. A retrospective, nonrandomized cohort study of different oxygen saturation policies in five neonatal units identified a lower incidence of ROP in the unit with the lowest oxygen saturation policy (14). In addition, a recent study demonstrated that changes in clinical practice where stricter controls of oxygen (and, therefore, presumably less fluctuations of oxygen) were implemented reduced the incidence of ROP considerably (34).

At birth, the physiologic systems in the human body rapidly alter to facilitate the transition from fetus to neonate. This normal process, however, may be subjected to conflicting messages when expected changes either do not occur, or occur at a different magnitude than that expected. Blood vessels in the retina can grow with apparent normal function after a preterm birth, and so a smooth transition from fetal to neonatal induction of vessel growth is possible. What level of relative hypoxia within the retina is required to prevent disordered growth is not yet clear.

Our model would suggest that variability around a mildly hypoxic mean enables normal growth of the retina, suggesting that nursing infants in slightly lower oxygen may reduce the incidence and severity of ROP. However, as this might compromise growth (35), brain growth, and maturation (36) and induce pulmonary hypertension (37), we would caution against the use of this data to alter oxygen supplementation policy. We concur with a recent commentary (38) that argues for a ran- domized controlled trial with long-term, subtle neurodevelopmental and respiratory outcomes as end points.

Acknowledgment. The authors thank Dr. Rob A. Elton for assistance with the statistical analysis.

\section{REFERENCES}

1. Fleck BW, Dangata Y 1994 Causes of visual handicap in the Royal Blind School, Edinburgh, 1991-2. Br J Ophthalmol 78:421

2. Gilbert C, Rahi J, Eckstein M, O'Sullivan J, Foster A 1997 Retinopathy of prematurity in middle-income countries. Lancet 350:12-14

3. Campbell K 1951 Intensive oxygen therapy as a possible cause of retrolental fibroplasia. A clinical approach. Med J Aust 2:48

4. Ashton N, Ward B, Serpell G 1954 Effect of oxygen on developing retinal vessels with particular reference to the problem of retrolental fibroplasia. Br J Ophthalmol 38:397-430

5. Patz A, Eastham A, Higginbotham DH, Kleh T 1953 Oxygen studies in retrolental fibroplasia. Am J Ophthalmol 36:1511-1522

6. Patz A 1982 The role of oxygen in retrolental fibroplasia. Am J Ophthalmol 94:715-743

7. McColm JR, Fleck BW 2001 Retinopathy of prematurity-causation. Semin Neonatol 6:453-460

8. Cunningham S, Fleck BW, Elton RA, McIntosh N 1995 Transcutaneous oxygen levels in retinopathy of prematurity. Lancet 346:1464-1465

9. Saito Y, Omoto T, Cho Y, Hatsukawa Y, Fujimura M, Takeuchi T 1993 The progression of retinopathy of prematurity and fluctuation in blood gas tension. Graefes Arch Clin Exp Ophthalmol 231:151-156

10. Reynaud X, Dorey KC 1994 Extraretinal neovascularization induced by hypoxic episodes in the neonatal rat. Invest Ophthalmol Vis Sci 35:3169-3177

11. Penn JS, Henry MM, Wall PT, Tolman BL 1995 The range of $\mathrm{PaO}_{2}$ variation determines the severity of oxygen induced retinopathy in newborn rats. Invest Ophthalmol Vis Sci 36:2063-2070

12. Cunningham S, McColm JR, Wade J, Sedowofia K, McIntosh N, Fleck BW 2000 A novel model of retinopathy of prematurity simulating preterm oxygen variability in the rat. Invest Ophthalmol Vis Sci 41:4275-4280

13. Mok JYQ, Hak H, McLaughin FJ, Pintar M, Canny GJ, Levison H 1988 Effect of age and state of wakefulness on transcutaneous oxygen values in preterm infants: a longitudinal study. J Pediatr 113:706-709

14. Tin W, Milligan DWA, Pennefather PM, Hey E 2001 Pulse oximetry, severe retinopathy, and outcome at one year in babies of less than 28 weeks gestation. Arch Dis Child Fetal Neonat Ed 84:106-110

15. Anderson CG, Benitz WE, Madan A 2002 Retinopathy of prematurity (ROP) and pulse oximetry: a national survey of recent practices. Pediatr Res 51:367A(abstr)

16. Stone J, Itin A, Alon T, Peer J, Gnessin H, Chan-Ling T, Keshet E 1995 Development of retinal vasculature is mediated by hypoxia-induced vascular endothelial growth factor (VEGF) expression by neuroglia. J Neurosci 15:4738-4747

17. McLeod DS, Brownstein R, Lutty GA 1996 Vaso-obliteration in the canine model of oxygen-induced retinopathy. Invest Ophthalmol Vis Sci 37:300-311

18. Smith LEH, Wesolowski E, McLellan A, Kostyk SK, D'Amato R, Sullivan R, D'Amore PA 1994 Oxygen induced retinopathy in the mouse. Invest Ophthalmol Vis Sci $35: 101-111$

19. McColm JR, Cunningham S 2000 The development of a computer controlled system to simulate in rats, the rapid, frequent changes in oxygen experienced by preterm infants developing retinopathy of prematurity. J Med Eng Technol 24:45-52

20. Cunningham S, Deere S, Elton RA, McIntosh N 1992 Neonatal physiological trend monitoring by computer. Int J Clin Monit Comput 9:221-227

21. Fetus and Newborn Committee of the AAP 1992 Clinical considerations in the use of oxygen. In: Freeman RK, Poland RL, Hauth JC, Merenstein GB (eds) Guidelines for Perinatal Care. American Colleges of Pediatrics/Obstetricians/Gynecologists, Elk Grove Village, IL

22. Holmes JM, Zhang S, Leske DA, Lanier WL 1998 Carbon-dioxide induced retinopathy in the neonatal rat. Curr Eye Res 17:608-616

23. Chan-Ling T 1997 Glial, vascular and neuronal cytogenesis in whole-mounted cat retina. Microsc Res Tech 36:1-16

24. Ricci B, Minicucci G, Manfredi A, Santo A 1995 Oxygen-induced retinopathy in the newborn rat: effects of hyperbarism and topical administration of timolol maleate. Graefes Arch Clin Exper Ophthalmol 233:226-230

25. Roberto KA, Tolman BL, Penn JS 1996 Long-term retinal vascular abnormalities in an animal model of retinopathy of prematurity. Curr Eye Res 15:932-937

26. Chan-Ling T, Gock B, Stone J 1995 The effect of oxygen on vasoformative cell division: evidence that 'physiological hypoxia' is the stimulus for normal retinal vasculogenesis. Invest Ophthalmol Vis Sci 36:1201-1214

27. Risau W 1997 Mechanisms of angiogenesis. Nature 386:671-674

28. Carmeliet P 2000 Mechanisms of angiogenesis and arteriogenesis. Nat Med 6:389395

29. Holmes JM, Duffner LA 1996 The effect of postnatal growth retardation on abnormal neovascularization in the oxygen exposed neonatal rat. Curr Eye Res 15:403-409

30. The STOP-ROP Multicenter Study Group 2000 Supplemental Therapeutic Oxygen for Prethreshold Retinopathy of Prematurity (STOP-ROP), a randomized, controlled trial. I: primary outcomes. Pediatrics 105:295-310

31. Phelps DL 1988 Reduced severity of oxygen-induced retinopathy in kittens recovered in $28 \%$ oxygen. Pediatr Res 24:106-109 
32. Chan-Ling T, Gock B, Stone J 1995 Supplemental oxygen therapy: basis for noninvasive treatment of retinopathy of prematurity. Invest Ophthalmol Vis Sci 36:1215-1230

33. McGregor ML, Bremer DL, Cole C, McClead RE, Phelps DL, Fellows RR, Oden N 2002 Retinopathy of prematurity outcome in infants with prethreshold retinopathy of prematurity and oxygen saturation $>94 \%$ in room air: the high oxygen percentage in retinopathy of prematurity study. Pediatrics 110:540-544

34. Chow LC, Wright KW, Sola A 2003 Can changes in clinical practice decrease the incidence of severe retinopathy of prematurity in very low birth weight infants? Pediatrics 111:339-345
35. Mortola JP, Lauzon A-M 1990 Body growth, lung and heart weight, and DNA content in newborn rats exposed to different levels of chronic hypoxia. Can J Physiol Pharmacol 68:1590-1594

36. Ment LR, Schwartz M, Makuch RW, Stewart WB 1998 Association of chronic sublethal hypoxia with ventriculomegaly in the developing rat brain. Brain Res Dev Brain Res 111:197-203

37. Keith IM, Tjen AL, Kraiczi H, Ekman R 2000 Three-week neonatal hypoxia reduces blood CGRP and causes persistent pulmonary hypertension in rats. Am J Physiol Heart Circ Physiol 279:H1571-H1578

38. Tin W 2002 Oxygen therapy: 50 years of uncertainty. Pediatrics 110:615-616 